\title{
Layered Reconstruction of Stippling Art
}

\author{
Amal Dev Parakkat \\ LIX, Ecole Polytechnique CNRS
}

\author{
Pooran Memari \\ LIX, Ecole Polytechnique CNRS
}

\author{
Marie-Paule Cani \\ LIX, Ecole Polytechnique CNRS
}
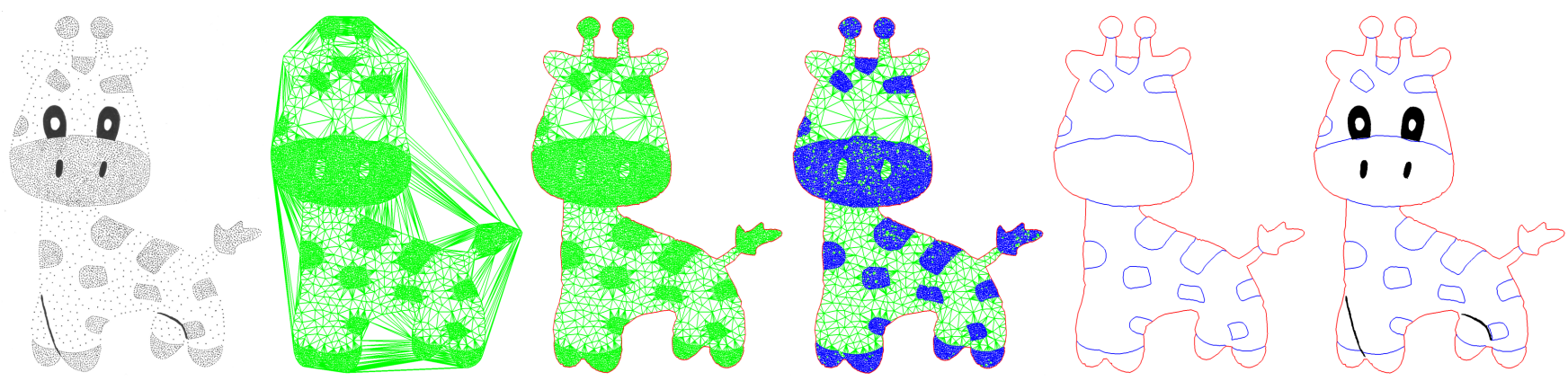

Figure 1: From left to right: Input stippled image, Delaunay triangulation of extracted stipples. Carving outer boundary. Trimming internal regions using $\alpha$-shape. Output layered boundaries without and with non-stipple regions.

\section{INTRODUCTION}

Given a point set $S \subseteq R^{2}$, reconstruction refers to the process of identifying a vector shape that best approximates the input. Although this field was pioneered since 1983 by Edelsbrunner [3] and has been heavily studied since then, the general problem still remains open, ill-posed and challenging. Solving it is essential for a wide range of applications, from image processing, pattern recognition and sketching to wireless networks.

The main motivation of our work is extend reconstruction to the case of stippled art (for eg. Hedcut drawings), as well as finding an inverse to the well studied problem of image conversion to blue noise (see for instance deGoes et al. [1]). Solution to this problem is extremely difficult since we expect reconstruction to be consistent with human cognition and perception of the art piece, while possible feature extraction heavily depends on various parameters of the input point set (density, distribution etc).

In this work, we introduce a dedicated variant of traditional 2D reconstruction in which the input point set (a stippled image) can include different regions corresponding to different point densities. Our method converts this input into a layered, vector representation, which includes a main shape plus internal, closed regions, defined by their boundary. Providing such structured output eases subsequent editing and processing, such as generating shaded vector images from the stippled input [6]. To achieve this, we first apply a layered reconstruction algorithm based on the detected regions in the input point set, and use the output for generating the outer and inner shape boundaries in the stippled drawing.

\section{PROBLEM AND CHALLENGES}

The problem can be formalized as follows: Let $R=\left\{R_{1}, R_{2}, \ldots, R_{n}\right\}$ be an image with regions $R_{1}, R_{2}, \ldots, R_{n}$, each region being represented by a set of points such that the adjacent regions have distinguishable point densities. Our goal is to extract a Layered reconstruction of this input, ie. to identify a set of regions defined by their boundary curves.
In this work, we only consider a simpler version of this problem, where the input point set has two distinct densities (region adjacency graph is two-colorable). We refer to these regions as low density and high density regions, respectively. We assume random point distributions on the input, since the latter can be hand-drawn.

Even in this simpler case, state of the art algorithms such as $\alpha$ shape [3] or the more recent $e c$-shape method $[4,5]$ cannot handle stippled input. Figure 2 shows the results of various algorithms on such input. Though the $\alpha$-shape is able to capture high density region with low $\alpha$ value, once we try to capture the region represented by low density points, the entire shape deteriorates (by filling the intended gaps between the high density regions; for example, the tail of goldfish shown in Figure 2). Whereas, random distribution of input points distorts the output of ec-shape algorithm. In the case of $\chi$-shape [2], the sculpting strategy based on edge length fails since the varying density cannot be taken into consideration. Also, the $\chi$-shape cannot identify inner boundaries. Curve reconstruction is a very similar related problem, where the samples are generated only from the object boundary. Curve reconstruction algorithm cannot be used if there are interior points (Figure 3 shows a sample result of a curve reconstruction algorithm [7] in this context).

Knowing that algorithms like $\alpha$-shape and $e c$-shape perform well in reconstructing shape from a point set with single density, the key idea behind the algorithm we propose, as the name suggests, is to apply reconstruction in a layered fashion to individually extract and reconstruct regions represented by similar point densities.

\section{PROPOSED APPROACH}

We have no assumptions on the density of the stipples in regions. Our algorithm takes only two parameters that could be estimated from region densities (one for maximum boundary edge length in the carving step and the other is the $\alpha$-shape parameter allowing to extract region boundaries in the trimming step). 

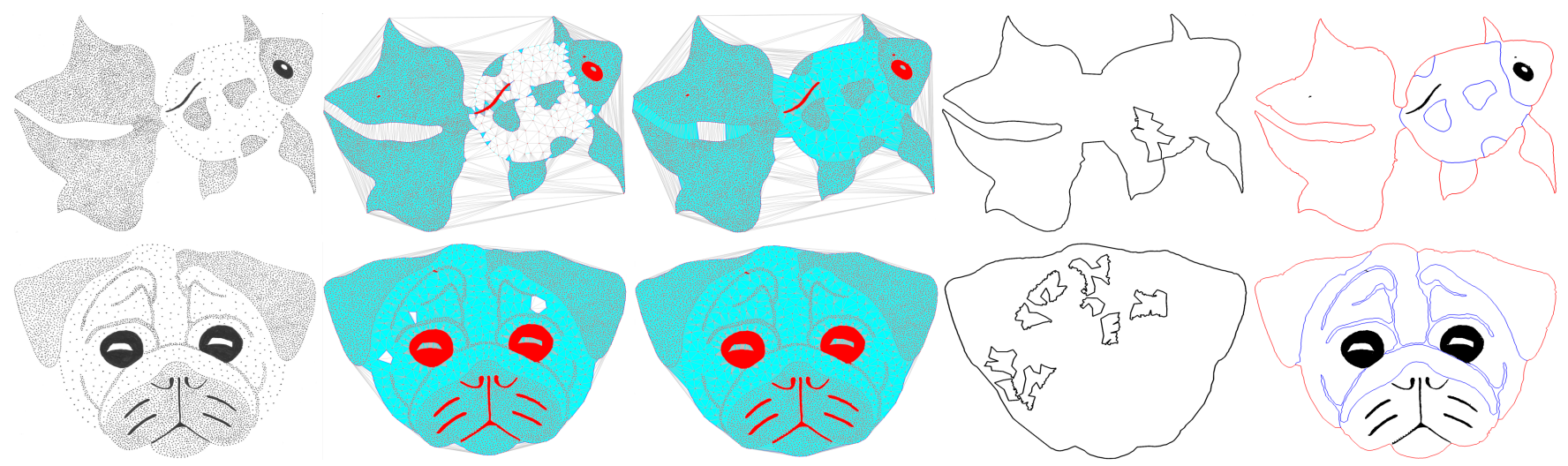

Figure 2: Comparison with existing methods. Left to right: Input stippled image, $\alpha$-shapes (for two $\alpha$ values), $e c$-shape and our result.

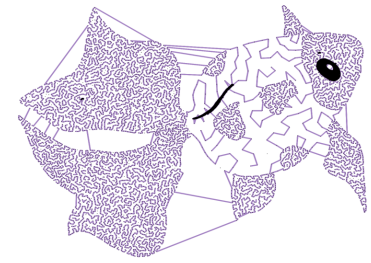

Figure 3: Result of a curve reconstruction

In carving step, we start from a Delaunay triangulation of the point set and progressively sculpt it as in the ec-shape method: starting from the exterior edges (edges not shared with any other triangle), edges are repeatedly removed until all the exterior edges satisfies the so-called diametric circle property (an edge $a b$ is sculpted only if the circle with $a b$ as a diametric chord is non empty). To avoid unnecessary blockages during sculpting, the algorithm removes an edge if its length exceeds a threshold (carving parameter).

Once the outer boundary (colored in red in Figure 1) is identified, trimming starts with the computation of $\alpha$-shape lying inside the carved result. If the parameter $\alpha$ is chosen between low and high density values, only points lying in high density regions will appear in the $\alpha$-shape. Therefore, extracting the boundary of $\alpha$-shape leads to an automatic reconstruction of inner boundaries between low and high density regions (colored in blue in Figure 1).

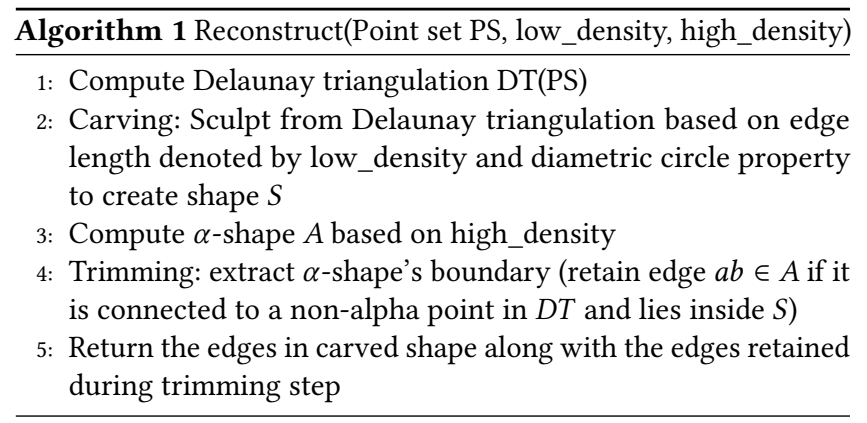

As an application, we used the proposed algorithm to reconstruct shape from stippled drawings, given as raster images. Initially, the pixels in the input image are clustered together based on its 8neighbourhood and each cluster is represented by a single point if it belongs to a stipple (so that non-stipple regions like eyes of the giraffe will be retained as such). The remaining non-stippled regions are separated before applying layered reconstruction and kept back after finding boundaries.

\section{FUTURE WORK}

Though the proposed algorithm requires two parameters (in order to automatically extract outer and inner boundaries between regions) we believe that they could be automatically computed based on local density estimation of the point set. This would be necessary for extending the method to the general case of stippled input with multiple densities, which is currently under development. Also, a complete system facilitating the creation of a shaded vector image from a stippled image, based on the point density and color information, is under progress.

\section{REFERENCES}

[1] Fernando de Goes, Katherine Breeden, Victor Ostromoukhov, and Mathieu Desbrun. 2012. Blue Noise Through Optimal Transport. ACM Trans. Graph. 31, 6, Article 171 (Nov. 2012), 11 pages.

[2] Matt Duckham, Lars Kulik, Mike Worboys, and Antony Galton. 2008. Efficient generation of simple polygons for characterizing the shape of a set of points in the plane. Pattern Recognition 41, 10 (2008), 3224 - 3236.

[3] H. Edelsbrunner, D. Kirkpatrick, and R. Seidel. 2006. On the Shape of a Set of Points in the Plane. IEEE Trans. Inf. Theor. 29, 4 (Sept. 2006), 551-559.

[4] Subhasree Methirumangalath, Shyam Sundar Kannan, Amal Dev Parakkat, and Ramanathan Muthuganapathy. 2017. Hole detection in a planar point set: An empty disk approach. Computers Graphics 66 (2017), 124 - 134. Shape Modeling International 2017.

[5] Subhasree Methirumangalath, Amal Dev Parakkat, and Ramanathan Muthuganapathy. 2015. A unified approach towards reconstruction of a planar point set. Computers Graphics 51 (2015), 90 - 97. Conference Shape Modeling International.

[6] Alexandrina Orzan, Adrien Bousseau, Holger Winnemöller, Pascal Barla, Joëlle Thollot, and David Salesin. 2008. Diffusion Curves: A Vector Representation for Smooth-Shaded Images. In ACM Transactions on Graphics (Proceedings of SIGGRAPH 2008), Vol. 27

[7] Amal Dev Parakkat and Ramanathan Muthuganapathy. 2016. Crawl Through Neighbors: A Simple Curve Reconstruction Algorithm. In Proceedings of the Symposium on Geometry Processing (SGP '16). 177-186. 